\title{
Diversity and evolution of the repetitive genomic content in Cannabis sativa
}

\author{
Rahul Pisupati ${ }^{1,3}$ (D), Daniela Vergara ${ }^{2}$ and Nolan C. Kane $2^{2^{*}}$
}

\begin{abstract}
Background: The repetitive content of the genome, once considered to be "junk DNA", is in fact an essential component of genomic architecture and evolution. In this study, we used the genomes of three varieties of Cannabis sativa, three varieties of Humulus lupulus and one genotype of Morus notabilis to explore their repetitive content using a graph-based clustering method, designed to explore and compare repeat content in genomes that have not been fully assembled.

Results: The repetitive content in the C. sativa genome is mainly composed of the retrotransposons LTR/Copia and LTR/Gypsy (14\% and 14.8\%, respectively), ribosomal DNA (2\%), and low-complexity sequences (29\%). We observed a recent copy number expansion in some transposable element families. Simple repeats and low complexity regions of the genome show higher intra and inter species variation.

Conclusions: As with other sequenced genomes, the repetitive content of $C$. sativa's genome exhibits a wide range of evolutionary patterns. Some repeat types have patterns of diversity consistent with expansions followed by losses in copy number, while others may have expanded more slowly and reached a steady state. Still, other repetitive sequences, particularly ribosomal DNA (rDNA), show signs of concerted evolution playing a major role in homogenizing sequence variation.
\end{abstract}

Keywords: Transposable elements, Next generation sequencing, Graph based clustering, Repeat explorer

\section{Background}

Repetitive sequences occupy the majority of any typical eukaryotic genome, yet are poorly understood in many respects. The effect of this repetitive content has been under debate for decades [1]. Numerous scientists regard it as parasitic or 'selfish DNA' [2]. Others think repetitive elements might play important roles in the host's genome by altering a gene's function [3], or by acting as raw material for new genes [4]. These ideas are not mutually exclusive, with repetitive sequences likely having both positive and negative effects in most genomes. Repetitive DNA elements can be mainly classified into two major groups based on their organization in the genome [5]. One group includes sequences showing a tandem repeat organization, where copies are arranged adjacently to each other, commonly (though not always) in or near centromeric and telomeric regions $[5,6]$. DNA elements which form

*Correspondence: Nolan.Kane@colorado.edu

${ }^{2}$ Ecology and Evolutionary Biology, University of Colorado, 80302 Boulder, USA Full list of author information is available at the end of the article tandem arrays such as satellite DNA, simple repeats and ribosomal DNA (rDNA) occur primarily in tandem repeat blocks [5, 7]. A second group of repetitive DNA sequences consist of elements which are dispersed across the whole genome [8]. These include mobile elements like DNA transposable elements (TEs) and retrotransposons such as long terminal repeat elements (LTRs), short interspersed nuclear elements (SINEs), and other dispersed repeats $[9,10]$.

Eukaryotic species show huge variation in genome size, ranging from only $2.3 \mathrm{Mb}$ in the microsporidian Encephalitozoon intestinalis [11] to over $152 \mathrm{~Gb}$ in the plant Paris japonica [12], five orders of magnitude of variation largely due to changes in repetitive content of the genome, although changes in gene content and ploidy also play a role [13]. Repetitive elements occupy a substantial fraction in most plant genomes, ranging from $20 \%$ in Arabidopsis thaliana to more than $80 \%$ in Helianthus annuus (sunflower) [14, 15]. Copy numbers of mobile elements range from thousands to millions per diploid 
genome [16]. Indeed, just within flowering plants, genome sizes differ roughly by 2500 -fold largely due to variation in the copy numbers of TEs and other highly repetitive sequences $[17,18]$.

The most common repeat sequences fall into several major categories. Due to their copy-paste transposition mechanism, active retrotransposons have the potential to increase their copy number affecting the genome size. LTR/Gypsy and LTR/Copia are two super-families of retrotransposons present in high copy numbers, resulting in major fractions in flowering plant's genomes [10]. Usually a smaller proportion of the genome, but highly important, ribosomal DNA (rDNA) encoding sequences in higher plants are arranged in long tandem arrays [7]. Plants typically have 500 to 40000 rDNA copies per diploid cell [19]. Simple sequence repeats, which exhibit high mutation rates, are also abundant within both animal and plant genomes [20]. Together, these classes of sequences make up the majority of the highcopy sequences in most well-characterized eukaryotic genomes.

Some classes of repetitive elements, such as LTR elements, provide an opportunity for deciphering the evolutionary demography of a family of retrotransposons within host genomes. Newly produced retrotransposons are $100 \%$ identical to the parental molecule but with no mechanism to maintain their homogeneity after insertion, they are expected to diverge neutrally [18]. Mutations gradually disfigure the elements to different lengths leading to an incomplete structure that might also inactivate them [21]. The magnitude of pairwise divergence between two LTRs can be used to infer their relative ages [22]. Studies on LTR families in rice [23], maize [22] and peas [24] calculated insertion ages using pairwise divergence among the elements and found that the average level of divergence for a large fraction of LTR elements is on the order of $1 \%$ or less [18]. These genomes thus are largely composed of recently duplicated repeat sequences.

Cannabis sativa (marijuana, hemp), a member of the family Cannabaceae is a widely cultivated plant with numerous genomic questions unanswered. The family Cannabaceae has 11 genera with approximately 100 species widely distributed throughout Asia and Europe [25]. Cannabis sativa is one of the earliest domesticated and cultivated plant species for fibre, oil, and for its medicinal and psychoactive properties [26]. Cannabis sativa has a diverse set of metabolic compounds as cannabinoids and terpenoids [27] and the numerous varieties vary morphologically in the production of these compounds [28]. Cannabis sativa Purple Kush (PK) is a commonly used recreational marijuana variety, while the cultivars 'Finola' and 'USO31' are hemp varieties used industrially [29]. These three varieties were sequenced in 2011 [30] and their sequences are publicly available at NCBI's short read archive. The estimated size of the haploid $C$. sativa genome is $830 \mathrm{Mb}$ and the draft genome assembly contains approximately $80 \%$ of the estimated genome [30]. In order to understand more about the genome of this important species, we studied its genomic diversity by exploring the TE dynamics.

Humulus lupulus (hops), the closest relative of C. sativa, has a genome three times as big (2570 Mb) [31]. Morus notabilis (mulberry), a closely related species to both $C$. sativa and $H$. lupulus diverged approximately 63.5 MYA [32] and was the closest related species of $C$. sativa sequenced until recently. The estimated genome size of $M$. notabilis is $357 \mathrm{Mb}$, which is less than half of C. sativa's genome [32]. Thus, these genera span a relatively large range of genome size variation. Using the genomes of these three related genera allows us to make comparisons between them to explain the variation in genome size within and among species of Cannabaceae family.

\section{Results}

\section{Characterizing repetitive content in genomes}

We determined that the repetitive content, characterized using Repeat Explorer (RE) [33]. Repeats occupy $64-65 \%$ of each C. sativa genome, $43 \%$ of the $M$. notabilis genome and $60.1 \%$ of the $H$. lupulus genome (Fig. 1). The analysis revealed LTR/Gypsy and LTR/Copia retrotransposons to be particularly abundant (ranging from $12-15 \%, 7-10 \%$ and $8-19 \%$ in C. sativa, M. notabilis, and H. lupulus respectively). LTR/Copia retrotransposons are mainly represented by Angela and AleII lineages, while LTR/Gypsy by Ogre/Tat, Athila and Chromovirus lineages. Simple and low complexity repeats were also found in high content (approximately 27\%, 14.3\% and 29\% in genomes of C. sativa, $M$. notabilis and $H$. lupulus respectively). rDNA occupies approximately $1.7-2.5 \%, 0.9 \%$ and $0.1 \%$ in C. sativa, $M$. notabilis, and $H$. lupulus genomes respectively (Additional file 1: Table S2). As a validation, we analyzed a well annotated genome of Arabidopsis thaliana using RE, which is consistent with our results (Additional file 1: Table S1). Also we included the genome repeat characterization of lineages in other Cannabis varieties, which are similar to those of PK, USO31, and Finola (Additional file 1: Table S1).

RE clusters sequences based on their pairwise similarity and generates graphs for each cluster using all-to-all pairwise comparisons. Each graph is similar to a de Brujjn graph [34] where every vertex correspond to a sequence, and their pairwise similarity score is expressed as edge weight [35]. The graph topology of the cluster can give information about the type of the repetitive element. In Fig. $2 \mathrm{a}$ and $\mathrm{b}$, graphs are less dense and have larger diameter which contains simple repeats and low complexity. Graphs with long units like LTR retrotransposons are characterized by the presence of multiple LTR domain 


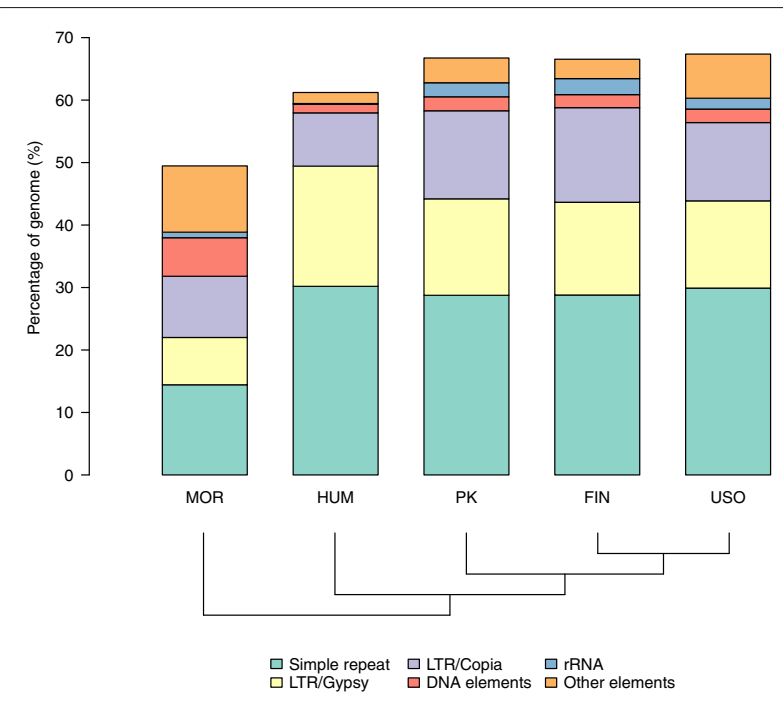

Fig. 1 Genome characterization by Repeat explorer. The graph based clustering algorithm (RE) characterized 64.5\%, 64.5\%, 65.2\%, 60.1\% and $43.3 \%$ of genome to be most high and medium copy number regions in M. notabilis (MOR), H. lupulus (HUM), C. sativa PK, Finola (FIN), and USO31 (USO), respectively. Cladogram modified from van Bakel et al. [30]

hits (Fig. 2c and d), which produce linear structures when the nodes connect densely between them into threadlike structures [35]. Full length LTR elements also produce circular layouts similar to the ones in Fig. 2c and they have annotated LTR domains.

Usually rDNA graphs exhibit a circular layout due to their tandem organisation [35]. However, our results (Fig. 2e and f) displayed densely connected linear arrangement which are due to the lack of sequencing coverage [35] at the ends of the rDNA repeats. This is likely due to variation in sequence among repeats as observed by
Novak et al. 2010 [35]. If that is the case, it suggests that the repeats are evolving concertedly across most of their length, but that repeats vary in the sequence of the spacer regions.

\section{Divergence calculations}

We performed a de novo assembly of sequencing reads in clusters using $R E$ that produced varying numbers of repeat family sequences in the different genotypes. We selected the sequences with a minimum length of 500 base pairs and subsequently filtered the library to remove plastid and virus sequences, which resulted in a repeat library (a set of sequences generated from highly repetitive genomic sequences) of 803 contigs in C. sativa PK, 548 in USO31, 854 in Finola, 424 contigs in H. lupulus and 396 contigs in M. notabilis. Coverages across the contigs, which measure the number of copies of a particular element present in the genome, range from as high of 500 to as low as 8 copies per genome in some repeats (Fig. 3). In order to understand whether the elements with more copies were more divergent, we plotted the average pairwise divergence between those contigs and their respective coverages (Fig. 3).

The pairwise divergence measure, $\theta_{\pi}$, is widely distributed in the repetitive sequences of $C$. sativa's genome varying from 0.11 to as low as 0 in terms of sequence divergence. The rDNA sequences present in the genome have very low pairwise divergence (average of 0.00327) with high coverages, occupying the top left portion in Fig. 3. This suggests rDNA sequences are conserved within the three varieties of $C$. sativa. On the other hand, simple repeats and low complexity found on the right portion in the plot have high pairwise divergences, which suggest that these sequences accumulate mutations at a higher rate.

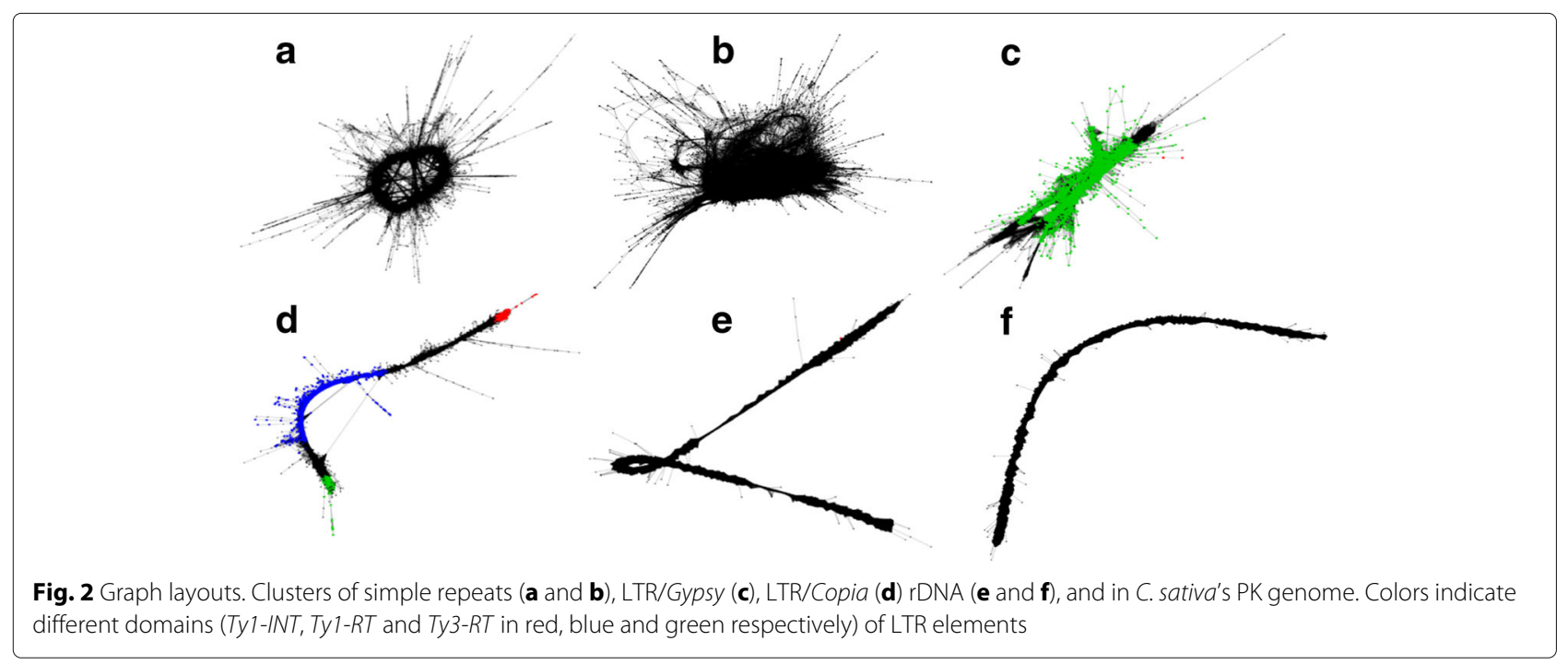



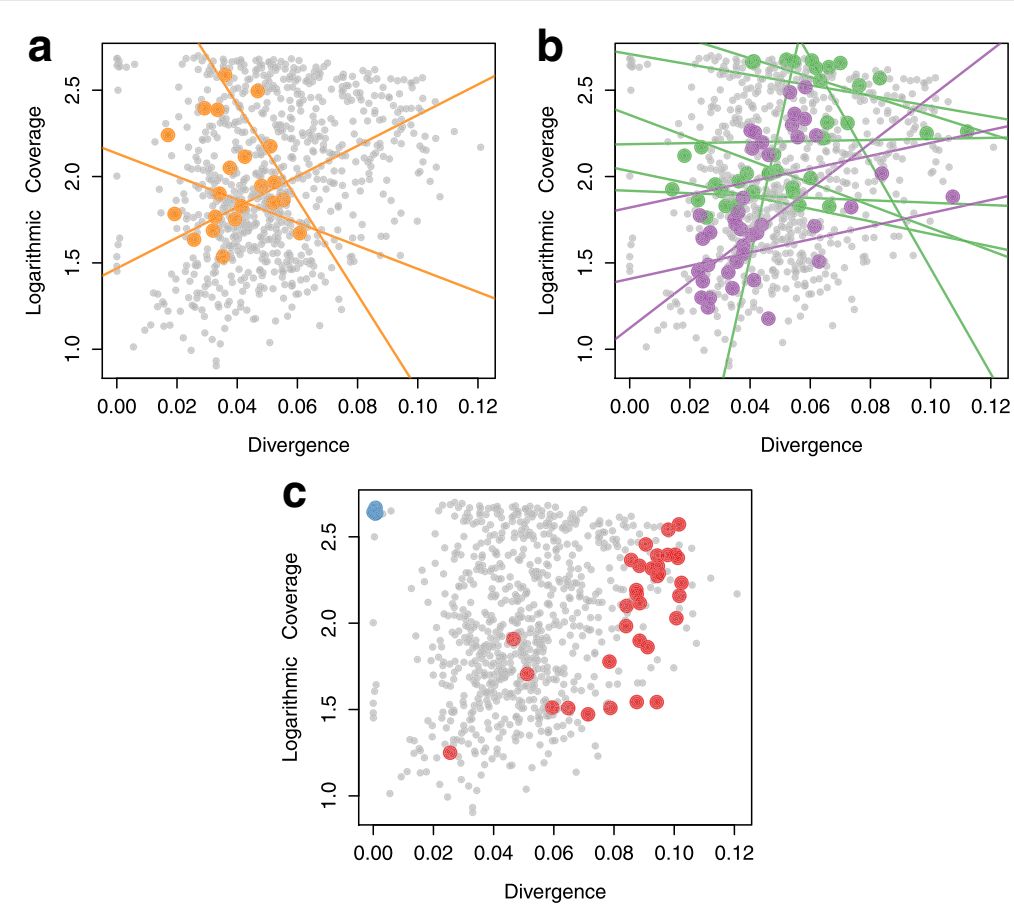

Fig. 3 Transposable elements in the PK genome and their amplification. Linear regresssions between the pairwise divergence of the TEs within the PK genome (x-axis), and each TE family's copy number (y-axis). Sequences clustered into the a LTR/Copia (orange), b LTR/Gypsy (Chromovirus and Athila in green and purple respectively), c rDNA (blue) and simple repeats (red) respectively. The remaining TEs in clusters are shown in grey dots

We calculated the estimated half-life for LTR elements present in clusters for C. sativa PK (PK), Finola (FIN), USO31 (USO), M. notabilis (MOR) and H. lupulus (HUM) (Fig. 4) as detailed in the methods section. We determined the median and standard deviation of the estimated half-life for LTR clusters in all the genomes. PK has the highest median $(0.1002$; s.d. $=0.331)$ of our analyzed genomes, followed by USO31 (0.0667; s.d. $=0.537)$, Finola $(0.0643$; s.d. $=0.351)$, H. lupulus $(0.0428$; s.d. $=$ $0.11)$, and $M$. notabilis $(0.0408$; s.d. $=0.14)$ in percentage sequence divergence. We also performed an ANOVA on the $\mathrm{R}$ statistical framework. The differences in the halflife among the genomes is non-significant $(\mathrm{F}=0.596, p$ $=0.67$ ). We used a Wilcox-Mann Whitney rank test to understand whether differences for each pair of genomes were significant. We found that PK differs from MOR $(0.049, p<0.05)$ but the difference is not significant compared to HUM $(0.035, p=0.1266)$, USO $(0.038, p=$ $0.0222)$ and FIN $(0.015, p=0.5376)$. Morus notabilis also showed significant differences from FIN $(-0.025, p=$ $0.0633)$ but not to USO $(-0.009, p=0.4082)$ or to HUM $(-0.007, p=0.6026)$.

\section{Network representations}

We described a detailed picture of the TE evolutionary history using the method AnTE [36], which also provides ancestral sequences. We show network representations of the relationships among the elements of LTR/Gypsy, LTR/Copia and for rDNA elements (Fig. 5). The LTR/Gypsy elements present in one of the clusters of the C. sativa PK genome, are shown in Fig. 5 a. For the LTR/Copia elements, most of the sequences (Fig. 5b) have a single ancestral sequence from which

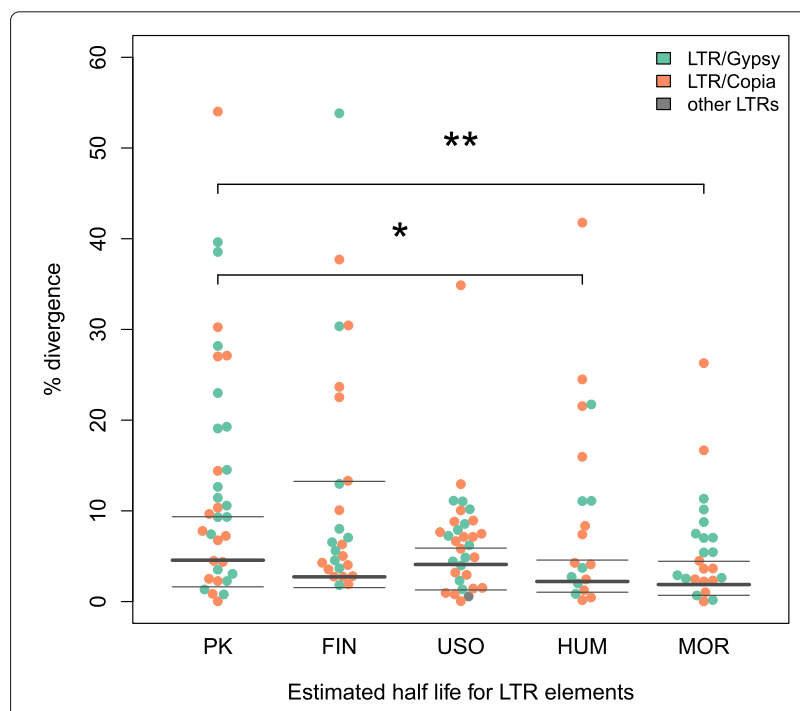

Fig. 4 LTR element divergence in the genomes. Median, 25th, and 75th percentiles of the estimated half-life for elements in clusters for C. sativa PK, Finola (FIN), USO31 (USO), H. lupulus (HUM) and $M$. notabilis (MOR). Asterisks mark significant difference (Wilcox - Mann Whitney Test, ${ }^{*}$ indicate $p$-value $\approx 0.1$ and ${ }^{* *}$ indicate $p$-value $<0.05$ ) 

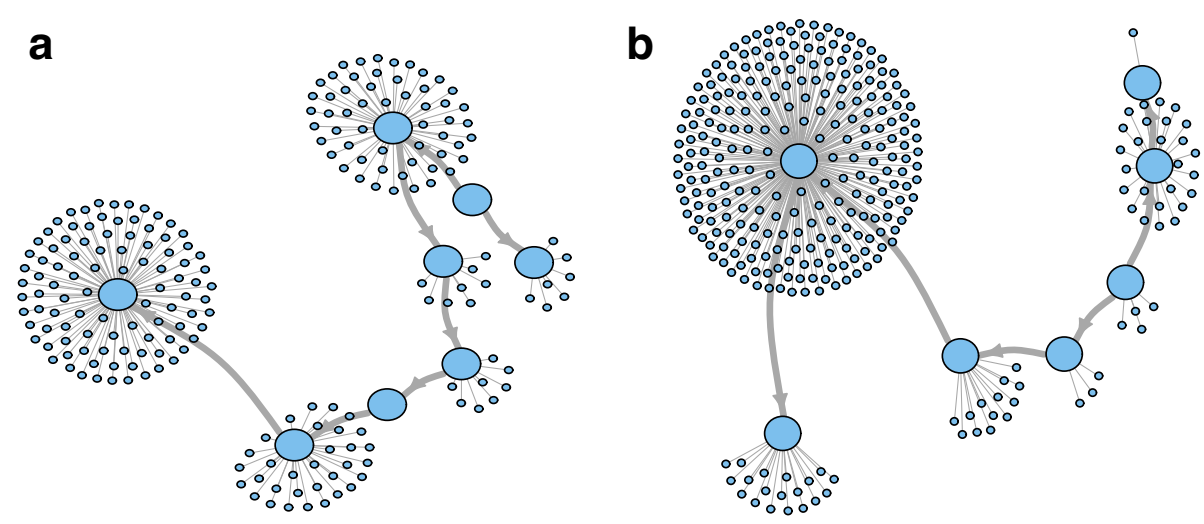

Fig. 5 Network representations using AnTE. LTR elements present in C. sativa PK genome represented in a phylogenetic network. All sequences are represented as nodes and their ancestor-descendant relationships are indicated by the arrows. a LTR/Gypsy. b LTR/Copia

populations diverged. This ancestral sequence is represented as a large circle from which arrows leave to form new sequences, suggesting a recent population expansions. In other words, after diverging from a single insertion event, each element proliferates to generate multiple copies of the same sequence in the genome.

\section{Comparative analysis among species}

Clustering analysis on a combined dataset of the five genomes shows that $11.54 \%, 9.81 \%, 9.41 \%, 6.42 \%$ of Finola, USO31, M. notabilis and H. lupulus genomes have equivalent clusters in the $C$. sativa PK genome respectively. We calculated the similarities in the repetitive content and across the TE families with respect to $C$. sativa $\mathrm{PK}$ genome.

It has been previously shown that $M$. notabilis and C. sativa diverged about 63.5 MYA, while Cannabis and Humulus diverged more recently [32]. Consistently, the repetitive sequence library in $M$. notabilis is the most divergent (Additional file 1: Table S3). The rDNA sequences are more conserved and their homologous copies in the genomes of M. notabilis, USO31, and Finola have divergences of $32 \%, 19 \%$, and $3 \%$ respectively. The paralogous copies of rDNA in the PK genome have an average divergence rate of $0.3 \%$, showing signs of concerted evolution. We also found that sequences for simple and low complexity repeats have considerable variation in percent similarity among the three species.

\section{Discussion}

Genome size variation can be partially explained by its repetitive content. We found that $64 \%$ of $C$. sativa genome is repetitive, which is less when compared to Maize ( $85 \%$ [37] and Sunflowers (80\% [38]) that have larger genomes, $2.3 \mathrm{~Gb}$ and $3.5 \mathrm{~Gb}$ respectively. However, despite substantial differences in genome size, proportions of repetitive content in species related to C. sativa are comparable, including H. lupulus (60.1\%), M. notabilis (47\% [32]), and $M$. domestica (67\% [39]). Thus, some differences in genome size are associated with quantifiable repeat content differences, but it is not the only component explaining genome size. Polyploidy, and smaller scale duplications also play a role [13] and these cannot be detected by RE. The repetitive content in $H$. lupulus is approximately $60.1 \%$, which is much lower compared to Maize and Sunflower that have bigger genomes, but smaller than C. sativa despite its the much larger genome size. These results, though surprising, are similar across multiple lineages within the species: $60.1 \%$ for $H$. lupulus Japanese wild hops, $61.3 \%$ for var. Lupulus, and $59.2 \%$ for var. cordifolius. The analysis of $A$. thaliana rules out artifacts from the pipeline (Additional file 1: Table S1). Additional, ongoing work, aims to better understand the repeat content in Humulus.

The majority of C. sativa's genome consists of LTR/Copia, LTR/Gypsy and simple repeats, unsurprisingly since LTR elements are present in high copy numbers in many flowering plant genomes [10]. The pairwise divergences across the elements can be used to calculate the insertion times of each TE family [9, 18, 23]. In Fig. 3, we show the logarithmic coverage (y-axis) against the pairwise divergence ( $x$-axis) across the elements, with the corresponding linear regressions for each cluster. Using the slope of the linear regressions, we estimated the halflife for the LTR elements in terms of sequence divergence. For some of the LTR/Gypsy elements, the half-life distributions suggest that $50 \%$ of the insertions are lost by the time they have diverged by an average of $8 \%$ at the nucleotide sequence level. On the other hand, LTR/Copia elements seem to survive much longer in the $C$. sativa genome, with an average half-life divergence of $15 \%$. LTR elements in other plants such as rice [40], maize [9] and wheat [41] seem to have lower half-life estimates, suggesting higher rates of turnover. The relatively high ages of 
LTR families in Cannabis may indicate a stable genomic content, with little recent turnover in repeat content.

In addition to estimating the half-life, the slope of the linear regressions in the divergence plot (Fig. 3) can reveal population dynamics and the type of selection present in the elements. A negative slope for the linear regression suggests that the element is under neutral selection $[9,18]$, because elements gradually accumulate mutations rendering them inactive. Some clusters that have a positive slope could either be under directional selection since they are not following a steady birth-death model. A positive slope can also signify a recent population expansion, meaning that sequences proliferate after diverging. This recent increase in copy number in a type of element can be confirmed with the phylogenetic relationship calculated through AnTE (Fig. 5b). The network analysis shows that many new insertions arose from an ancestral sequence for some LTR/Gypsy elements in the C. sativa genome. Since we already know that some of these repeat families are unique to the derived Y chromosome in Cannabis [42-44], it will be interesting to investigate the functional significance, if any, of other repeat families, particularly as genetic maps and other resources become better developed in this species.

Relating transposon sequences and copy number to phenotypic traits is often challenging. However, it is known that in Cannabis, retroposons are associated with sex differences among males and females [42-44]. The analysis presented here is solely on females, but ongoing work on additional genotypes will further explore repeat content differences among males and females, as well as investigating copy number associations with other important traits, such as the production of secondary metabolites (cannabinoids) many of them with medicinal properties [45, 46].

In most eukaryotes, rDNA genes are present in tandem repeated arrays in high copy numbers [47]. The copy number of rDNA genes varies between species, and in $A$. thaliana approximately $10 \%$ of the genome size variation is due to the differences in rDNA gene copies [48]. We found that $2 \%$ of the $C$. sativa's genome is composed of rDNA genes (Additional file 1: Table S2). We also found that $H$. lupulus has approximately $0.1 \%$ of its genome occupied by rDNA genes, with fewer copies despite its larger genome size. Unsurprisingly, we found that rDNA elements present in the $C$. sativa genome show signs of concerted evolution, as expected based on substantial work in other species [49-51]. However, a handful of other repeat sequences also showed similar levels of concerted evolution (Fig. 3).

The other major type of repeats found in the noncoding part of the genome are simple sequence repeats, microsatellites or low-complexity regions [52], and they occupy approximately $25 \%$ of C. sativa's genome, $13 \%$ of
M. notabilis genome, and $30 \%$ of $H$. lupulus genome. Since we estimated the repeat content from raw sequence reads, our estimate is unbiased. Additionally, our estimate of these repetitive elements in $M$. notabilis genome is consistent with previous findings [32]. These sequences show high divergence across species, as expected considering the fact that these regions have higher mutation rates [52].

\section{Conclusions}

Our study gives insight into the composition and the dynamics of repeats in the genomes of three varieties of $C$. sativa and two related genera. Among the three genera, we found similar repetitive content. The prevalence of LTR/Copia and LTR/Gypsy elements in the three genomes of $C$. sativa and $H$. lupulus is higher than in M. notabilis, which may partially explain the variation in the genome sizes. Our estimated half-life for LTR/Copia elements is higher compared to LTR/Gypsy elements in the $C$. sativa genome, showing higher turnover in these elements. rDNA elements, as well as some other unclassified repeats, show signs of concerted evolution, and some LTR elements show recent population expansion. Finally, the network representations for the repetitive elements present in genome validate the population expansion in the LTR elements, and help to explain the proliferation of these major components in the genomes.

\section{Methods}

\section{Determining repetitive content}

We analyzed the raw genomic reads obtained from the published genome of three different $C$. sativa varieties PK, USO31, and Finola [30], mulberry (M. notabilis) [32] and hops (H. lupulus) [31], using a graph based clustering pipeline. This method requires high-throughput genome sequencing data and does not require an assembled genome for characterizing the repetitive content. Genome shotgun sequencing reads were taken from NCBI's Short Read Archive (SRA) [SRR352164], [SRR351494], [SRR351929], [SRR847535] and [DRR024456] for the three $C$. sativa varieties, $M$. notabilis and $H$. lupulus respectively. To confirm the repeat content estimates for $H$. lupulus, which were surprisingly low given the large genome size of the species, we also analyzed Japanese wild hop, var. Lupulus and var. cordifolius within SRA database [DRR024456], [DRR024410], [DRR024452]. We also analyzed four other varieties of C. sativa (NCBI accession numbers SRR3294442, SRR3294438, SRR3294431, and SRR3294475 (Additional file 1: Table S1) [53], and a genome of $A$. thaliana [SRR519656] [48] using the same pipeline, to validate the pipeline.

We did trimming and quality filtering of the raw reads using the fastx-toolkit with a quality threshold of 30 and minimum read length of 80 . Reduced sets of randomly selected genomic reads for the four genomes ( $1 \mathrm{x}$ coverage) 
were separately subjected to clustering using RE [33] with default parameters. We used RepBase libraries (accessed 31 January 2014) of Viridiplantae and Conserved Domain Database, which contains protein domains derived from plant mobile genetic elements to annotate and classify repeat family of clusters.

\section{Pairwise divergence between elements}

$\mathrm{RE}$ is a graph based clustering algorithm, where the sequences are clustered based on their similarity. RE produces contigs by assembling sequences from each cluster that serve as reference sequences and represent TE elements present in genome. We established a library with consensus contigs of each repeat class form each genome, with a minimum sequence length of $500 \mathrm{bp}$. We annotated these contigs using RepeatMasker and Repeat library (accessed 31 January, 2014). We used BurrowsWheeler Aligner (bwa) [54] to align the genomic reads back to these contigs with default parameters. We calculated the average pairwise divergence for sequences aligned in each contig using Popoolation [55] from a pileup file generated using SAMtools (version 0.1.15) with default parameters [56]. We determined average depth across the contig from the pileup file using custom perl scripts (hosted on GitHub, https://github.com/rbpisupati/ ExploringTEsinCannabisGenome/), which is considered to be the copy number of that particular TE family present in the cluster. We calculated the half-life of TEs as measured by sequence divergence (as a proxy for age), assuming a steady-state birth-death model. The half-life is a linear function with the slope of a linear regression line in a log-arithmetic plot between the pairwise divergence and the copy number. We performed a linear regression analysis (lm), a Wilcox - Mann - Whitney analysis, and a t-test in the R statistical framework (version 3.1) with default parameters.

\section{Phylogenetic networks}

Approximately eight million sequence reads in each genome were aligned to the consensus repeat library. Based on the TE annotation of the contigs, we extracted reads aligned to a position in the contig. In order to infer the dynamics and TE ancestry, we used a Bayesian method, AnTE [36] on those aligned sequences with default parameters. AnTE reconstructs the ancestral relationships among the sequences.

\section{Divergence across the species}

To establish the differences between the species, we performed an inter-species comparative analysis of the genomes using RE. We identified homologous sequences between the repeat library of each pair of genomes using NCBI BLAST [57]. We also determined the divergence for TE families across the genomes using the percent similarity from BLAST [57].

\section{Additional file}

\begin{abstract}
Additional file 1: Table S1. Raw data information and repeat content for different varieties of Cannabis sativa, Humulus lupulus, Morus notabilis and Arabidopsis thaliana. Table S2. Repetitive content (percentage of the genome) in the genomes of C. sativa PK, Finola (FIN), USO31 (USO), $\mathrm{H}$. lupulus (HUM) and, M. notabilis (MOR). Table S3. Repeat sequence conservation, as measured by percent sequence similarity among consensus sequences from each repeat class in each genotype, compared to the consensus repeats from the C. sativa PK genome. The missing entries are due to the absence of specific families in either of the genomes' repeat libraries. (PDF $163 \mathrm{~kb}$ )
\end{abstract}

\section{Abbreviations}

HUM: Humulus lupulus; LTR: Long terminal repeat; MOR: Morus notabilis; MYA: Million years ago PK: Cannabis sativa purple kush variety; RE: Repeat explorer; rDNA: Ribosomal DNA; TE: Transposable elemen; USO: Cannabis sativa USO31

\section{Acknowledgements}

We would like to thank A. Wachholder for help with the phylogenetic analysis; S. Tittes and K. Keepers for analysis help, and discussion; P Mitra for discussions about the sequence analysis.

\section{Funding}

This research was supported by donations to the University of Colorado Foundation gift fund 13401977-Fin8 to NCK and by HRD, Government of India to RP.

\section{Availability of data and materials}

The scripts and datasets generated during the current study are hosted in the public GitHub repository (https://github.com/rbpisupati/

ExploringTEsinCannabisGenome/).

\section{Authors' contributions}

$\mathrm{RP}$ analyzed the data and wrote the first draft of the manuscript. DV and NCK directed the project, contributed to the analysis and manuscript revisions. All authors read and approved the final manuscript.

\section{Ethics approval and consent to participate}

The whole genome sequencing data for the germplasm of C. sativa, H. lupulus and M. notabilis were taken from publicly available NCBI SRA database.

\section{Consent for publication}

Not applicable

\section{Competing interests}

The authors declare that they have no competing interests.

\section{Publisher's Note}

Springer Nature remains neutral with regard to jurisdictional claims in published maps and institutional affiliations.

\section{Author details}

${ }^{1}$ Department of Biotechnology, Indian Institute of Technology, 721302 Kharagpur, India. ${ }^{2}$ Ecology and Evolutionary Biology, University of Colorado, 80302 Boulder, USA. ${ }^{3}$ Present address: Gregor Mendel Institute, Dr. Bohr-gasse 3, 1030 Vienna, Austria.

Received: 30 March 2017 Accepted: 24 January 2018

Published online: 21 February 2018

References

1. Haubold B, Wiehe T. How repetitive are genomes?. Bmc Bioinformatics. 2006;7(1):541.

2. Doolittle WF, Sapienza C. Selfish genes, the phenotype paradigm and genome evolution. Nature. 1980;284(5757):601-3.

3. Martin A, Troadec C, Boualem A, Rajab M, Fernandez R, Morin H, Pitrat M, Dogimont C, Bendahmane A. A transposon-induced epigenetic change leads to sex determination in melon. Nature. 2009;461(7267):1135-8. 
4. Zhou L, Mitra R, Atkinson PW, Hickman AB, Dyda F, Craig NL. Transposition of hat elements links transposable elements and $v(d) j$ recombination. Nature. 2004:432(7020):995-1001.

5. Kubis S, Schmidt T, Heslop-Harrison JSP. Repetitive dna elements as a major component of plant genomes. Ann Bot. 1998;82(suppl 1):45-55

6. Tautz D, Renz M. Simple sequences are ubiquitous repetitive components of eukaryotic genomes. Nucleic Acids Res. 1984;12(10):4127-38.

7. Rogers SO, Bendich AJ. Ribosomal rna genes in plants: variability in copy number and in the intergenic spacer. Plant Mol Biol. 1987;9(5):509-20.

8. Jurka J, Kapitonov W, Kohany O, Jurka MV. Repetitive sequences in complex genomes: structure and evolution. Annu Rev Genomics Hum Genet. 2007:8:241-59.

9. SanMiguel P, Tikhonov A, Jin YK, Motchoulskaia N, et al. Nested retrotransposons in the intergenic regions of the maize genome. Science. 1996;274(5288):765.

10. Kumar A, Bennetzen JL. Plant retrotransposons. Annu Rev Genet. 1999:33(1):479-532

11. Corradi N, Pombert JF, Farinelli L, Didier ES, Keeling PJ. The complete sequence of the smallest known nuclear genome from the microsporidian Encephalitozoon intestinalis. Nat Commun. 2010;1:77.

12. Pellicer J, Fay MF, Leitch IJ. The largest eukaryotic genome of them all? Botanical J Linnean Soc. 2010;164(1):10-15.

13. Wendel JF, Jackson SA, Meyers BC, Wing RA. Evolution of plant genome architecture. Genome Biol. 2016;17(1):37.

14. Initiative $A G$, et al. Analysis of the genome sequence of the flowering plant arabidopsis thaliana. Nature. 2000;408(6814):796.

15. Gill N, Buti M, Kane N, Bellec A, Helmstetter N, Berges H, Rieseberg LH. Sequence-based analysis of structural organization and composition of the cultivated sunflower (helianthus annuus I.) genome. Biology. 2014;3(2):295-319.

16. Elder Jr JF, Turner BJ. Concerted evolution of repetitive dna sequences in eukaryotes. Q Rev Biol. 1995;70(3):297-320.

17. Proost S, Pattyn P, Gerats T, Van de Peer Y. Journey through the past: 150 million years of plant genome evolution. Plant J. 2011;66(1):58-65.

18. Lynch M, Walsh B. The origins of genome architecture. Sunderland: Sinauer Associates; 2007.

19. Ingle J, Timmis JN, Sinclair J. The relationship between satellite deoxyribonucleic acid, ribosomal ribonucleic acid gene redundancy, and genome size in plants. Plant Physiol. 1975;55(3):496-501.

20. Smỳkal P, Kalendar R, Ford R, Macas J, Griga M. Evolutionary conserved lineage of angela-family retrotransposons as a genome-wide microsatellite repeat dispersal agent. Heredity. 2009;103(2):157-67.

21. Caspi A, Pachter L. Identification of transposable elements using multiple alignments of related genomes. Genome Res. 2006;16(2):260-70.

22. SanMiguel $P$, Gaut BS, Tikhonov A, Nakajima Y, Bennetzen JL. The paleontology of intergene retrotransposons of maize. Nat Genet. 1998;20(1):43-5.

23. Ma J, Devos KM, Bennetzen JL. Analyses of Itr-retrotransposon structures reveal recent and rapid genomic dna loss in rice. Genome Res. 2004;14(5): 860-9.

24. Jing R, Knox MR, Lee JM, Vershinin AV, Ambrose M, Ellis TN, Flavell AJ. Insertional polymorphism and antiquity of pdr1 retrotransposon insertions in pisum species. Genetics. 2005;171(2):741-52.

25. Wills S. Cannabis use and abuse by man: an historical perspective. The Genus Cannabis; 1998, p. 1.

26. Measham F, Newcombe $R$, Parker $H$. The normalization of recreational drug use amongst young people in north-west england. Br J Sociol. 1994;45(2):287-312.

27. Marks MD, Tian L, Wenger JP, Omburo SN, Soto-Fuentes W, He J, Gang DR, Weiblen GD, Dixon RA. Identification of candidate genes affecting $\delta 9$-tetrahydrocannabinol biosynthesis in cannabis sativa. J Exper Botan. 2009;60:3715-26.

28. Flores-Sanchez IJ, Verpoorte R. Pks activities and biosynthesis of cannabinoids and flavonoids in cannabis sativa I. plants. Plant Cell Physiol. 2008;49(12):1767-82.

29. Datwyler SL, Weiblen GD. Genetic variation in hemp and marijuana (cannabis sativa I.) according to amplified fragment length polymorphisms. J Forensic Sci. 2006;51(2):371-5.

30. Van Bakel H, Stout JM, Cote AG, Tallon CM, Sharpe AG, Hughes TR, Page JE. The draft genome and transcriptome of cannabis sativa. Genome Biol. 2011;12(10):102.
31. Natsume S, Takagi H, Shiraishi A, Murata J, Toyonaga H, Patzak J, Takagi M, Yaegashi H, Uemura A, Mitsuoka C, et al. The draft genome of hop (humulus lupulus), an essence for brewing. Plant Cell Physiol. 2014;56: 428-41.

32. He N, Zhang C, Qi X, Zhao S, Tao Y, Yang G, Lee TH, Wang X, Cai Q, Li D, Lu M. Draft genome sequence of the mulberry tree Morus notabilis. Nat Commun. 2013;4:2445.

33. Novák P, Neumann P, Pech J, Steinhaisl J, Macas J. Repeatexplorer: a galaxy-based web server for genome-wide characterization of eukaryotic repetitive elements from next-generation sequence reads. Bioinformatics. 2013;29(6):792-3.

34. Zerbino DR, Birney E. Velvet: algorithms for de novo short read assembly using de bruijn graphs. Genome Res. 2008;18(5):821-9.

35. Novák P, Neumann P, Macas J. Graph-based clustering and characterization of repetitive sequences in next-generation sequencing data. BMC Bioinformatics. 2010;11(1):378.

36. Wacholder AC, Cox C, Meyer TJ, Ruggiero RP, Vemulapalli V, Damert A, Carbone L, Pollock DD. Inference of transposable element ancestry. PLoS Genet. 2014;10(8):1004482.

37. Schnable PS, Ware D, Fulton RS, Stein JC, Wei F, Pasternak S, Liang C, Zhang J, Fulton L, Graves TA, et al. The b73 maize genome: complexity, diversity, and dynamics. Science. 2009;326(5956):1112-5.

38. Staton SE, Bakken BH, Blackman BK, Chapman MA, Kane NC, Tang S, Ungerer MC, Knapp SJ, Rieseberg LH, Burke JM. The sunflower (helianthus annuus I.) genome reflects a recent history of biased accumulation of transposable elements. Plant J. 2012;72(1):142-53.

39. Velasco R, Zharkikh A, Affourtit J, Dhingra A, Cestaro A, Kalyanaraman A Fontana P, Bhatnagar SK, Troggio M, Pruss D, et al. The genome of the domesticated apple (malus [times] domestica borkh). Nat Genet. 2010;42(10):833-9.

40. Vitte C, Panaud O. Formation of solo-ltrs through unequal homologous recombination counterbalances amplifications of Itr retrotransposons in rice oryza sativa I. Mol Biol Evol. 2003;20(4):528-40.

41. SanMiguel PJ, Ramakrishna W, Bennetzen JL, Busso CS, Dubcovsky J. Transposable elements, genes and recombination in a $215-\mathrm{kb}$ contig from wheat chromosome 5a m. Funct Integr Genomics. 2002;2(1):70-80.

42. Sakamoto K, Ohmido N, Fukui K, Kamada H, Satoh S. Site-specific accumulation of a line-like retrotransposon in a sex chromosome of the dioecious plant cannabis sativa. Plant Mol Biol. 2000;44(6):723-32

43. Sakamoto K, Abe T, Matsuyama T, Yoshida S, Ohmido N, Fukui K, Satoh S Rapd markers encoding retrotransposable elements are linked to the male sex in cannabis sativa I. Genome. 2005:48(5):931-6.

44. Razumova OV, Alexandrov OS, Divashuk MG, Sukhorada TI, Karlov GI. Molecular cytogenetic analysis of monoecious hemp (cannabis sativa I.) cultivars reveals its karyotype variations and sex chromosomes constitution. Protoplasma. 2016;253(3):895-901.

45. Russo EB. Taming thc: potential cannabis synergy and phytocannabinoid-terpenoid entourage effects. Br J Pharmacol. 2011;163(7):1344-64.

46. Volkow ND, Baler RD, Compton WM, Weiss SR. Adverse health effects of marijuana use. N Engl J Med. 2014;370(23):2219-27.

47. Prokopowich CD, Gregory TR, Crease TJ. The correlation between rdna copy number and genome size in eukaryotes. Genome. 2003;46(1):48-50.

48. Long Q, Rabanal FA, Meng D, Huber CD, Farlow A, Platzer A, Zhang Q, Vilhjálmsson BJ, Korte A, Nizhynska $\mathrm{V}$, et al. Massive genomic variation and strong selection in arabidopsis thaliana lines from sweden. Nat Genet. 2013;45(8):884-90.

49. Kane N, Sveinsson S, Dempewolf H, Yang JY, Zhang D, Engels JM, Cronk Q. Ultra-barcoding in cacao (theobroma spp.; malvaceae) using whole chloroplast genomes and nuclear ribosomal dna. Am J Botany. 2012;99(2):320-9.

50. Schaal BA, Learn Jr GHL. Ribosomal dna variation within and among plant populations. Ann Mo Bot Gard. 1988;1207-16.

51. Liao D. Concerted evolution: molecular mechanism and biological implications. Am J Hum Genet. 1999:64(1):24-30.

52. Ellegren H. Microsatellites: simple sequences with complex evolution. Nat Rev Genet. 2004;5(6):435-5.

53. Lynch RC, Vergara D, Tittes S, White K, Schwartz C, Gibbs MJ, Ruthenburg TC, deCesare K, Land DP, Kane NC. Genomic and chemical diversity in cannabis. Crit Rev Plant Sci. 2016;35(5-6):349-63. 
54. Li H, Durbin R. Fast and accurate long-read alignment with burrows-wheeler transform. Bioinformatics. 2010;26(5):589-95.

55. Kofler R, Orozco-terWengel P, De Maio N, Pandey RV, Nolte V, Futschik A Kosiol C, Schlötterer C. Popoolation: a toolbox for population genetic analysis of next generation sequencing data from pooled individuals. PLoS ONE. 2011;6(1):15925.

56. Li H, Handsaker B, Wysoker A, Fennell T, Ruan J, Homer N, Marth G, Abecasis $G$, Durbin R, et al. The sequence alignment/map format and samtools. Bioinformatics. 2009;25(16):2078-9.

57. Altschul SF, Gish W, Miller W, Myers EW, Lipman DJ. Basic local alignment search tool. J Mol Biol. 1990;215(3):403-10.

Submit your next manuscript to BioMed Central and we will help you at every step:

- We accept pre-submission inquiries

- Our selector tool helps you to find the most relevant journal

- We provide round the clock customer support

- Convenient online submission

- Thorough peer review

- Inclusion in PubMed and all major indexing services

- Maximum visibility for your research

Submit your manuscript at www.biomedcentral.com/submit
C Biomed Central 